\title{
On a product of two formational tcc-subgroups*
}

\author{
A. Trofimuk \\ Communicated by L. A. Kurdachenko
}

For the 70th anniversary of L. A. Kurdachenko

\begin{abstract}
A BS TR ACT. A subgroup $A$ of a group $G$ is called tcc-subgroup in $G$, if there is a subgroup $T$ of $G$ such that $G=A T$ and for any $X \leqslant A$ and $Y \leqslant T$ there exists an element $u \in\langle X, Y\rangle$ such that $X Y^{u} \leqslant G$. The notation $H \leqslant G$ means that $H$ is a subgroup of a group $G$. In this paper we consider a group $G=A B$ such that $A$ and $B$ are tcc-subgroups in $G$. We prove that $G$ belongs to $\mathfrak{F}$, when $A$ and $B$ belong to $\mathfrak{F}$ and $\mathfrak{F}$ is a saturated formation of soluble groups such that $\mathfrak{U} \subseteq \mathfrak{F}$. Here $\mathfrak{U}$ is the formation of all supersoluble groups.
\end{abstract}

\section{Introduction}

Throughout this paper, all groups are finite and $G$ always denotes a finite group. We use the standard notations and terminology of $[1,2]$. The monographs $[2,3]$ contain the necessary information of the theory of formations.

We say that the subgroups $A$ and $B$ of a group $G$ are totally permutable if every subgroup of $A$ is permutable with every subgroup of $B$.

Asaad and Shaalan [4] proved the supersolubility of a group $G=A B$ such that the subgroups $A$ and $B$ are totally permutable and supersoluble, see [4, Theorem 3.1]. Following Maier [5] such factorization is called

*This work was supported by the BRFFR (grant No. F19RM-071).

2010 MSC: 20D10.

Key words and phrases: supersoluble group, totally permutable product, saturated formation, tcc-permutable product, tcc-subgroup. 
totally permutable product of the subgroups $A$ and $B$. In the same paper Maier showed that this statement is also true for the saturated formations containing the formation $\mathfrak{U}$ of all supersoluble groups.

Theorem 1 ([5, Theorem]). Let $G=H K$ be the totally permutable product of the subgroups $H$ and $K$. Let $\mathfrak{F}$ be a saturated formation such that $\mathfrak{L} \subseteq \mathfrak{F}$. If $H$ and $K$ lie in $F$, then so does $G$. Here $\mathfrak{L}$ denote the class of groups all of whose Sylow subgroups are cyclic.

In [5] Maier also proposes the following question: «Does the above result extend to non-saturated formations which contain all supersoluble groups?»

Ballester-Bolinches and Perez-Ramos gave a positive answer to this question in [6].

Theorem 2 ([6, Theorem]). Let $\mathfrak{F}$ be a formation containing the class $\mathfrak{U}$ of all supersoluble groups. Suppose that $G=H K$ be the totally permutable product of the subgroups $H$ and $K$. If $H$ and $K$ belong to $\mathfrak{F}$, then $G$ belongs to $\mathfrak{F}$.

In works [7], [8] the authors extended a previous Maier's result by considering an arbitrary number of pairwise totally permutable factors.

In the articles [9]-[13] (see also the references from [13]) we can see that the supersolubility of a group can also be obtained for other generalizations of totally permutable product.

The notation $H \leqslant G$ means that $H$ is a subgroup of a group $G$. So, for example, the product $G=A B$ is said to be tcc-permutable [13], if for any $X \leqslant A$ and $Y \leqslant B$ there exists an element $u \in\langle X, Y\rangle$ such that $X Y^{u} \leqslant G$. The subgroups $A$ and $B$ in this product are called tcc-permutable.

One of the main results of [12] for two tcc-permutable factors is formulated as follows.

Theorem 3 ([12, Theorem 5]). Let $\mathfrak{F}$ be a saturated formation containing $\mathfrak{U}$. Let the group $G=H K$ be the tcc-permutable product of subgroups $H$ and $K$. If $H \in \mathfrak{F}$ and $K \in \mathfrak{F}$, then $G \in \mathfrak{F}$.

Besides, in [12] the authors gave examples showing that Theorem 3 not remains true for arbitrary non-saturated formations containing $\mathfrak{U}$ even in the universe of soluble groups.

Now we introduce the following concept

Definition 1. A subgroup $A$ of a group $G$ is called tcc-subgroup in $G$, if it satisfies the following conditions: 
1) there is a subgroup $T$ of $G$ such that $G=A T$;

2) for any $X \leqslant A$ and $Y \leqslant T$ there exists an element $u \in\langle X, Y\rangle$ such that $X Y^{u} \leqslant G$.

Clear that by condition 2 of Definition $1, G=A T$ is the tcc-permutable product of the subgroups $A$ and $T$. In this case, we say that the subgroup $T$ is a tcc-supplement to $A$ in $G$.

If $G=A B$ is the tcc-permutable product of subgroups $A$ and $B$, then the subgroups $A$ and $B$ are tcc-subgroups in $G$. The converse is false.

Example 1. Let $Z_{n}$ be a cyclic group of order $n$. Dihedral group $G=$ $\langle a\rangle \rtimes\langle c\rangle,|a|=12,|c|=2$ ([14], IdGroup=[24,6]) is the product of tcc-subgroups $A=\left\langle a^{3} c\right\rangle$ of order 2 and $B=\left\langle a^{10}\right\rangle \rtimes\langle c\rangle$ of order 12 . But $A$ and $B$ are not tcc-permutable. Indeed, there are the subgroups $X=A$ and $Y=\langle c\rangle$ of $A$ and $B$ respectively such that doesn't exist $u \in\langle X, Y\rangle=\left\langle a^{3}\right\rangle \rtimes\langle c\rangle$ such that $X Y^{u} \leqslant G$.

In the present paper we prove the following theorem.

Theorem A. Let $G=A B$, where $A$ and $B$ are tcc-subgroups in $G$. Let $\mathfrak{F}$ be a saturated formation of soluble groups such that $\mathfrak{U} \subseteq \mathfrak{F}$. Suppose that $A$ and $B$ belong to $\mathfrak{F}$. Then $G$ belongs to $\mathfrak{F}$.

\section{Preliminaries}

In this section, we give some definitions and basic results which are essential in the sequel.

A group whose chief factors have prime orders is called supersoluble. If $H \leqslant G$ and $H \neq G$, we write $H<G$. The notation $H \unlhd G$ means that $H$ is a normal subgroup of a group $G$. Denote by $Z(G), F(G)$ and $\Phi(G)$ the centre, Fitting and Frattini subgroups of $G$ respectively, and by $\mathrm{O}_{p}(G)$ the greatest normal $p$-subgroup of $G$. Denote by $\pi(G)$ the set of all prime divisors of order of $G$. The semidirect product of a normal subgroup $A$ and a subgroup $B$ is written as follows: $A \rtimes B$. If $H$ is a subgroup of $G$, then $H_{G}=\bigcap_{x \in G} H^{x}$ is called the core of $H$ in $G$.

A formation $\mathfrak{F}$ is said to be saturated if $G / \Phi(G) \in \mathfrak{F}$ implies $G \in \mathfrak{F}$. Let $\mathbb{P}$ be the set of all prime numbers. A formation function is a function $f$ defined on $\mathbb{P}$ such that $f(p)$ is a, possibly empty, formation. A formation $\mathfrak{F}$ is said to be local if there exists a formation function $f$ such that $G \in \mathfrak{F}$ if and only if for any chief factor $H / K$ of $G$ and any $p \in \pi(H / K)$, one has $G / C_{G}(H / K) \in f(p)$. We write $\mathfrak{F}=L F(f)$ and $f$ is a local definition of $\mathfrak{F}$. By [3, Theorem IV.3.7], among all possible local definitions of a local 
formation $\mathfrak{F}$ there exists a unique $f$ such that $f$ is integrated (i.e. $f(p) \subseteq \mathfrak{F}$ for all $p \in \mathbb{P}$ ) and full (i.e. $f(p)=\mathfrak{N}_{p} f(p)$ for all $p \in \mathbb{P}$ ). Here $\mathfrak{N}_{p}$ is the formation of all $p$-groups. Such local definition $f$ is said to be canonical local definition of $\mathfrak{F}$. By [3, Theorem IV.4.6], a formation is saturated if and only if it is local.

If a group $G$ contains a maximal subgroup $M$ with trivial core, then $G$ is said to be primitive and $M$ is its primitivator.

Lemma 1. Let $\mathfrak{F}$ be a saturated formation and $G$ be a group. Assume that $G \notin \mathfrak{F}$, but $G / N \in \mathfrak{F}$ for all non-trivial normal subgroups $N$ of $G$. Then $G$ is a primitive group.

Proof. Since $\mathfrak{F}$ is a saturated formation, it follows that $\Phi(G)=1$ and $G$ contains a unique minimal normal subgroup $N$. For some maximal subgroup $M$ of $G$, we have $G=N M$, because $\Phi(G)=1$. It is obvious that the core $M_{G}=1$. Hence $G$ is a primitive group.

Lemma 2 ([1, Theorem II.3.2]). Let $G$ be a soluble primitive group and $M$ is a primitivator of $G$. Then the following statements hold:

(1) $\Phi(G)=1$;

(2) $F(G)=C_{G}(F(G))=O_{p}(G)$ and $F(G)$ is an elementary abelian subgroup of order $p^{n}$ for some prime $p$ and some positive integer $n$;

(3) $G$ contains a unique minimal normal subgroup $N$ and moreover, $N=F(G)$;

(4) $G=F(G) \rtimes M$ and $O_{p}(M)=1$;

Lemma 3 ([13, Theorem 1, Propositions 1, 2]). Let $G=A B$ be the tcc-permutable product of subgroups $A$ and $B$ and $N$ be a minimal normal subgroup of $G$. Then the following statements hold:

(1) $\{A \cap N, B \cap N\} \subseteq\{1, N\}$;

(2) if $N \leqslant A \cap B$ or $N \cap A=N \cap B=1$, then $|N|=p$, where $p$ is a prime.

Lemma 4 ([12, Theorem 4]). Let $G=A B$ be the tcc-permutable product of subgroups $A$ and $B$. Then $[A, B] \leqslant F(G)$.

Lemma 5. Let $A$ be a tcc-subgroup in $G$ and $Y$ be a tcc-supplement to $A$ in $G$. Then the following statements hold:

(1) $A$ is a tcc-subgroup in $H$ for any subgroup $H$ of $G$ such that $A \leqslant H$;

(2) $A N / N$ is a tcc-subgroup in $G / N$ for any $N \unlhd G$;

(3) for every $A_{1} \unlhd A$ and $X \leqslant Y$ there exists an element $y \in Y$ such that $A_{1} X^{y} \leqslant G$. In particular, $A_{1} M \leqslant G$ for some maximal subgroup $M$ 
of $Y$ and $A_{1} H \leqslant G$ for some Hall $\pi$-subgroup $H$ of soluble $Y$ and any $\pi \subseteq \pi(G)$

(4) $A_{1} K \leqslant G$ for every subnormal subgroup $K$ of $Y$ and for every $A_{1} \unlhd A ;$

(5) if $T \unlhd G$ such that $T \leqslant A$ and $T \cap Y=1$, then $T_{1} \unlhd G$ for every $T_{1} \unlhd A$ such that $T_{1} \leqslant T$;

(6) if $T \unlhd G$ such that $T \cap A=1$ and $T \leqslant Y$, then $A_{1} \leqslant N_{G}\left(T_{1}\right)$ for every $T_{1} \unlhd T$ and for every $A_{1} \unlhd A$.

Proof. 1. Since $Y$ is a tcc-supplement to $A$ in $G$, it follows that $G=A Y$, $A$ and $Y$ are tcc-permutable subgroups of $G$. By Dedekind's identity, $H=H \cap A Y=A(H \cap Y)$. Since $H \cap Y \leqslant Y$, then for any $X \leqslant A$ and $Z \leqslant H \cap Y$ there exists an element $u \in\langle X, Z\rangle$ such that $X Z^{u} \leqslant G$. Hence $A$ and $H \cap Y$ are tcc-permutable and therefore $A$ is a tcc-subgroup in $H$.

2. Since $G=A Y$, we have $G / N=(A N / N)(Y N / N)$. Let $B / N$ be an arbitrary subgroup of $A N / N$ and $X / N$ be an arbitrary subgroup of $Y N / N$. Since $N \leqslant B \leqslant A N$, it follows that by Dedekind's identity, $B=B \cap A N=(B \cap A) N$. Similarly, $X=X \cap Y N=(X \cap Y) N$. Since $B \cap A \leqslant A$ and $X \cap Y \leqslant Y$, we have $(B \cap A)(X \cap Y)^{u} \leqslant G$ for some $u \in\langle B \cap A, X \cap Y\rangle$. Hence

$$
(B / N)(X / N)^{u N}=(B \cap A)(X \cap Y)^{u} N / N \leqslant G / N
$$

for $u N \in\langle B \cap A, X \cap Y\rangle N / N \subseteq\langle B, X\rangle N / N=\langle B / N, X / N\rangle$. Thus $A N / N$ is a tcc-subgroup in $G / N$.

3. Since $A$ is a tcc-subgroup in $G$, by definition, for every $A_{1} \unlhd A$ and $X \leqslant Y$ there exists an element $u \in\left\langle A_{1}, X\right\rangle$ such that $A_{1} X^{u} \leqslant G$. Because $u \in G=A Y=Y A$, it follows that $u=y a$ for some $y \in Y$ and $a \in A$. Then

$$
A_{1} X^{u}=A_{1} X^{y a}=A_{1}\left(X^{y}\right)^{a}=A_{1}^{a}\left(X^{y}\right)^{a}=\left(A_{1} X^{y}\right)^{a} \leqslant G .
$$

Hence there is a subgroup $A_{1} X^{y}$ in $G$ for some $y \in Y$. Clearly, that if $X$ is a Hall $\pi$-subgroup of $Y$, then $H=X^{y}$ is a Hall $\pi$-subgroup of $Y$. Thus $A_{1} H \leqslant G$. Similarly, for maximal subgroup $X$ of $Y$. Then $M=X^{y}$ is a maximal subgroup of $Y$ and $A_{1} M \leqslant G$.

4. Since $K$ is subnormal in $Y$, there is a chain of subgroups $Y=$ $K_{0} \geqslant K_{1} \geqslant \ldots \geqslant K_{n-1} \geqslant K_{n}=K$ such that $K_{i+1}$ is normal in $K_{i}$ for all $i$. We use induction by $n$. By (3), there exists an element $y \in Y$ such that $A_{1} K_{1}^{y}=A_{1} K_{1} \leqslant G$. Hence the statement holds for $n=0$ and $n=1$. Therefore $n \geqslant 2$. By (1), $A$ is a tcc-subgroup in $A K_{1}$ and $K_{1}$ is a 
tcc-supplement to $A$ in $A K_{1}$. Since the length of subnormal chain between $K$ and $K_{1}$ less than $n$, it follows that by induction, there is a subgroup $A_{1} K$ of $A K_{1}$. Consequently $A_{1} K \leqslant G$.

5. By (3), there is a subgroup $T_{1} Y$ of $G$. Since $T_{1}=T \cap T_{1} Y$ is normal in $T_{1} Y$, we have $Y \leqslant N_{G}\left(T_{1}\right)$ and $T_{1}$ is normal in $G=A Y$.

6. Since $T_{1}$ is subnormal in $Y$, it follows that by (4), there is a subgroup $A_{1} T_{1}$ of $G$ for any $A_{1} \unlhd A$. Because $T_{1}=T \cap A_{1} T_{1}$ is normal in $A_{1} T_{1}$, we have $A_{1} \leqslant N_{G}\left(T_{1}\right)$.

Lemma 6. Let $A$ be a tcc-subgroup in a soluble primitive group $G$ and $Y$ be a tcc-supplement to $A$ in $G$. Suppose that $N$ is a minimal normal subgroup of $G$. If $N \cap A=1$ and $N \leqslant Y$, then $A$ is cyclic of order dividing $p-1$.

Proof. Since $N \cap A=1$ and $N \leqslant Y$, by Lemma $5(6), A \leqslant N_{G}(K)$ for any $K \unlhd N$. Since $N$ is an elementary abelian group, it follows that $|N|=p^{s}$ for some prime $p$ and some integer $s$, and $N=N_{1} \times N_{2} \times \ldots \times N_{s}$, where $N_{i}=\left\langle x_{i}\right\rangle$ and $\left|N_{i}\right|=p$. Because $A \leqslant N_{G}\left(N_{i}\right)$, we have $A$ induces a power automorphism group on $N$. Indeed, let $a \in A$ and $x_{i} \in N_{i}, x_{j} \in N_{j}$, $i \neq j$. Suppose that $x_{i}^{a}=x_{i}^{t}, x_{j}^{a}=x_{j}^{r}$ and $\left(x_{i} x_{j}\right)^{a}=\left(x_{i} x_{j}\right)^{m}=x_{i}^{m} x_{j}^{m}$, because $N$ is abelian. Then $x_{i}^{m} x_{j}^{m}=x_{i}^{t} x_{j}^{r}$. Consequently $m=t=r$ and a transforms every element of $N$ to the same power. By [2, Theorem 2.3], $A / C_{A}(N) \simeq \leqslant \mathrm{P}(N)$, where $\mathrm{P}(N)$ is the power automorphism group of $N$. Since $C_{G}(N)=N$, it follows that $C_{A}(N)=1$. On the other hand, $\mathrm{P}(N)$ is a cyclic group of order $p-1$. Really $\mathrm{P}(N)$ is a group of scalar matrices over the field $\mathbf{P}$ consisting of $p$ elements. Hence $\mathrm{P}(N)$ is isomorphic to the multiplicative group $\mathbf{P}^{*}$ of $\mathbf{P}$ and besides, $\mathbf{P}^{*}$ is a cyclic group of order $p-1$. Therefore $A$ is a cyclic group of order dividing $p-1$.

Lemma 7 ([15, Lemma 2.16]). Let $\mathfrak{F}$ be a saturated formation containing $\mathfrak{U}$ and $G$ be a group with a normal subgroup $E$ such that $G / E \in \mathfrak{F}$. If $E$ is cyclic, then $G \in \mathfrak{F}$.

\section{Proof of Theorem A}

Assume that the claim is false and let $G$ be a minimal counterexample. Let $N$ be a non-trivial normal subgroup of $G$. The quotients $A N / N \simeq$ $A / A \cap N$ and $B N / N \simeq B / B \cap N$ are tcc-subgroup in $G / N$ by Lemma 5 (2), $A N / N \simeq A / A \cap N \in \mathfrak{F}$ and $B N / N \simeq B / B \cap N \in \mathfrak{F}$, because $\mathfrak{F}$ is a formation. Hence the quotient $G / N=(A N / N)(B N / N) \in \mathfrak{F}$ by induction. 
If $F(G) \neq 1$, then $G / F(G) \in \mathfrak{F}$ and consequently $G$ is soluble, since $\mathfrak{F}$ is a formation of soluble groups. Hence $F(G)=1$ and by Lemma 4, $Y \leqslant C_{G}(A)$. Then $A$ is normal in $G$ and $G$ is soluble.

Since $\mathfrak{F}$ is saturated, it follows that $G$ is primitive by Lemma 1 . Hence $\Phi(G)=1, N=C_{G}(N)=F(G)=\mathrm{O}_{p}(G)$ is a unique minimal normal subgroup of $G$ by Lemma 2 and $G=N \rtimes M$, where $|N|=p^{n}$ and $M$ is a primitivator.

By Lemma 3, is either $|N|=p$, or $N \leqslant A$ and $N \cap Y=1$, or $N \cap A=1$ and $N \leqslant Y$. In the first case, by Lemma $7, G \in \mathfrak{F}$. Suppose that $N \leqslant A$ and $N \cap Y=1$. Since $Y$ is a tcc-subgroup in $G$, it follows that by Lemma 6, $Y$ is a cyclic group of order dividing $p-1$. Then $Y \in g(p)$, where $g$ is the canonical local definition of $\mathfrak{U}$. Since $\mathfrak{U} \subseteq \mathfrak{F}$, we have by [3, Proposition IV.3.11], $g(p) \subseteq f(p)$, where $f$ is the canonical local definition of $\mathfrak{F}$. Hence $Y \in f(p)$.

Because $Y$ is a cyclic group of order dividing $p-1$, it follows that $Y$ is contained in some Hall $p^{\prime}$-subgroup $H$ of $G$. Hence there exists an element $g \in G$ such that $Y \leqslant H=H_{1}^{g} \leqslant M^{g}$, where $H_{1}$ is a Hall $p^{\prime}$-subgroup of $G$ such that $H_{1} \leqslant M$, because $|G: M|=p^{n}$. Let $M_{1}=M^{g}$. Then $G=N \rtimes M_{1}$ and $M_{1}$ is a primitivator. Clearly that $M_{1}=\left(A \cap M_{1}\right) Y$.

Since $N \leqslant A$, we have $A=N \rtimes\left(A \cap M_{1}\right)$. Because $A \in \mathfrak{F}$, it follows that $A / C_{A}\left(N_{1}\right) \in f(p)$, where $N_{1}$ is a minimal normal subgroup of $A$ such that $N_{1} \leqslant N$. Since $A$ is a tcc-subgroup in $G$, by Lemma $5(5)$, $N_{1}$ is normal in $G$. Hence $N=N_{1}$ and $C_{A}\left(N_{1}\right)=C_{A}(N)=N$. Then $A \cap M_{1} \simeq A / N \in f(p)$.

We consider the direct product $\left(A \cap M_{1}\right) \times Y=\left\{(a, b), a \in A \cap M_{1}, b \in\right.$ $Y$ \}. Let $\varphi:\left(A \cap M_{1}\right) \times Y \rightarrow M_{1}=\left(A \cap M_{1}\right) Y$ be a function and $\varphi(a, b)=$ $a b$. Since by Lemma $4,[A, Y] \leqslant F(G)=N$, it follows that $\left[A \cap M_{1}, Y\right] \leqslant N$. Because $\left[A \cap M_{1}, Y\right] \leqslant M_{1}$, we have $\left[A \cap M_{1}, Y\right] \leqslant M_{1} \cap N=1$. Hence $A \cap M_{1} \leqslant C_{M_{1}}(Y)$ and $\varphi$ is an epimorphism. Then by [2, Theorem 2.3],

$$
\left(A \cap M_{1}\right) \times Y / \operatorname{Ker} \varphi \simeq \operatorname{Im} \varphi=M_{1} .
$$

Since $f(p)$ is a formation, $A \cap M_{1} \in f(p)$ and $Y \in f(p)$, it follows that $M_{1} \in f(p)$. Because $N \in \mathfrak{N}_{p}$, we have $G \in \mathfrak{N}_{p} f(p)=f(p) \subseteq \mathfrak{F}$.

So, we assume that $N \cap A=1$ and $N \leqslant Y$. Similarly, we can show that $N \cap B=1$ and $N \leqslant X$, where $X$ is a tcc-supplement to $B$ in $G$. By Lemma 6, $A$ and $B$ are cyclic. Hence $G$ is supersoluble and therefore $G \in \mathfrak{F}$.

The theorem is proved. 
Corollary 1. Let $A$ and $B$ be tcc-subgroups in $G$ and $G=A B$. If $A$ and $B$ are supersoluble, then $G$ is supersoluble.

Corollary 2 ([4, Theorem 3.1]). Suppose that $A$ and $B$ are supersoluble subgroups of $G$ and $G=A B$. Suppose further that $A$ and $B$ are totally permutable. Then $G$ is supersoluble.

\section{References}

[1] B. Huppert, Endliche Gruppen I. Berlin-Heidelberg-New York, Springer, 1967.

[2] V.S. Monakhov, Introduction to the Theory of Final Groups and Their Classes [in Russian]. Vysh. Shkola, Minsk, 2006.

[3] K. Doerk and T. Hawkes, Finite soluble groups. Berlin-New York: Walter de Gruyter, 1992.

[4] M. Asaad, A. Shaalan, On the supersolubility of finite groups, Arch. Math., 53, 1989, pp.318-326.

[5] R. Maier, A completeness property of certain formations, Bull. Lond. Math. Soc. 24, 1992, pp.540-544.

[6] A. Ballester-Bolinches, M. D. Perez-Ramos, A question of R. Maier concerning formations, J. Algebra, 182, 1996, pp.738-747.

[7] A. Carocca, A note on the product of F-subgroups in a finite group, Proc. Edinb. Math. Soc., 39, 1996, pp.37-42.

[8] A. Ballester-Bolinches, M. C. Pedraza-Aguilera, M. D. Pérez-Ramos, Finite groups which are products of pairwise totally permutable subgroups, Proc. Edinb. Math. Soc., 41, 1998, pp. 567-572.

[9] W. Guo, K. P. Shum, A. N. Skiba, Criterions of supersolubility for products of supersoluble groups, Publ. Math. Debrecen, 68(3-4), 2006, pp.433-449.

[10] M. Arroyo-Jorda, P. Arroyo-Jorda, A. Martinez-Pastor, M. D. Perez-Ramos, On finite products of groups and supersolubility, J. Algebra, 323, 2010, pp.2922-2934.

[11] M. Arroyo-Jorda, P. Arroyo-Jorda, M. D. Perez-Ramos, On conditional permutability and saturated formations, Proc. Edinb. Math. Soc., 54, 2011, pp.309-319.

[12] M. Arroyo-Jorda, P. Arroyo-Jorda, A. Martinez-Pastor and M. D. Perez-Ramos, On conditional permutability and factorized groups, Annali di Matematica Pura ed Applicata, 193, 2014, pp.1123-1138.

[13] M. Arroyo-Jorda, P. Arroyo-Jorda, Conditional permutability of subgroups and certain classes of groups, Journal of Algebra, 476, 2017, pp.395-414.

[14] GAP, Groups, Algorithms, and Programming, Version 4.10.1. www.gap-system.org, 2019.

[15] A. N. Skiba, On weakly s-permutable subgroups of finite groups, J. Algebra, 315, 2007, pp.192-209.

\section{CONTACT INFORMATION}

Alexander Trofimuk Department of Mathematics, Gomel Francisk Skorina State University, Gomel 246019, Belarus

E-Mail(s): alexander.trofimuk@gmail.com

Received by the editors: 03.06.2019. 